\title{
Position statement of the Menopause Society of Sri Lanka on estrogen and progestogen use in peri- and post-menopausal women and some other management options
}

\author{
M D P Gooneratne ${ }^{1}$, Hemantha Perera ${ }^{2}$
}

Sri Lanka Journal of Obstetrics and Gynaecology 2009; 31: 128-135

\section{Contents}

\section{Estrogen and progestogen}

Introduction

Interpreting Studies

\section{Management considerations}

Pretreatment evaluation

Vasomotor symptoms

Urogenital and Vaginal symptoms

The role of local vaginal estrogen for treatment of vaginal atrophy in postmenopausal women

Progestogen indication

Venous thromboembolism

Stroke

Diabetes mellitus

Breast cancer

Endometrial cancer

Ovarian cancer

Colorectal cancer

Gallbladder disease

Osteoporosis

Dementia and cognitive decline

Depression

Premature menopause and premature ovarian failure

Lower doses

Non-oral therapy

Long-term therapy in symptomatic women

Symptom recurrence

Indications for extended use

Quality of life

\footnotetext{
${ }^{1}$ Consultant Obstetrician and Gynaecologist, Founder President, Menopause Society of Sri Lanka.

${ }^{2}$ Consultant Obstetrician and Gynaecologist, Sri Jayewardenepura General Hospital.

Corresponding author: M D Gooneratne

E-mail:pem_deva@sltnet.lk
}

Biological identity of products

Continuous versus sequential EPT

Conclusions

Summary practice points

Alternative and complementary therapies

Tibolone

Phytoestrogens

Herbalism

Summary practice points

The role of testosterone therapy in postmenopausal women

The role of calcium in peri- and post-menopausal women

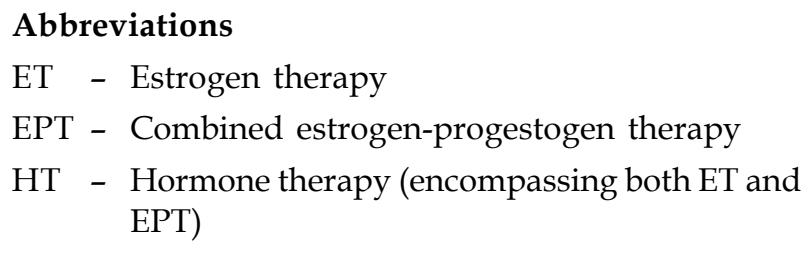

\section{Introduction}

Since the release of the position statement of the Menopause Society of Sri Lanka (MSSL) in 2008, there were many new findings and views on the Women's Health Initiative (WHI) and the Million Women Study (MWS). This has clarified some of the uncertainties about the role of HT among health professionals and women. This resulted in the release of this revised position statement by the MSSL.

\section{Biological identity of products}

Systemic HRT administered as EPT has the two principal components, estrogen and progestogen. Both hormones may be natural or artificial in constitution. Therefore it may be of clinical relevance to discuss specific drug classes, but the results from large epidemiological studies are in general assumed to apply to all systemic HRT regimens regardless of hormonal structure or route of delivery. 
The principal difference between oral and nonoral (transdermal, subcutaneous, nasal) route of administration is the first-pass metabolism in the gut and liver changing markedly the pharmacokinetics of the hormonal compound.

As estrogen, natural estradiol valerate or micronized 17- $\beta$ estradiol is often used in Europe whereas the conjugated equine estrogen (CEE) derived from pregnant mare's urine is the preferred product in the US.

After oral intake the equipotent dose of $2 \mathrm{mg} 17-\beta$ estradiol corresponds to approximately $0.625 \mathrm{mg}$ of conjugated estrogens and to $50 \mu \mathrm{g}$ delivered by patches or $1.5 \mathrm{mg}$ given as percutaneous gel.

Progesterone is not available from any natural source without extraction and synthesis. In contrast to progesterone, the progestogens used in HRT are all rapidly absorbed after oral intake. The most common products are those based on the C19 steroid testosterone (i.e norethisterone, levonorgestrel), the 17hydroxyprogesterone derivatives (i.e. medroxyprogesterone acetate (MPA), megestrol acetate), 19norprogesterone derivatives (i.e. nomegestrol, promegestone, trimegestone) or the retroprogesterone, dydrogesterone. Drosperinone which is a derivative of spironolactone, has the added advantage of preventing weight gain and possible beneficial effects on hypertension.

It is not possible to extrapolate conclusions from the study of one compound, dose, and route of administration directly to another. However, in the absence of clinical trial data for each estrogen and progestogen, the clinical trial results for one agent may be generalized to all agents within the same family.

\section{Continuous versus sequential EPT}

The recent RCTs were conducted with continuous combined regimens. Cohort and case-control studies do not allow conclusions in favour of any regimen with regard to the breast. Concerning the cardiovascular risk, no information is available for this comparison, and there are no additional data from other RCTs comparing other relevant clinical endpoints during systemic sequential versus continuous combined EPT.

\section{Indication for progestogens}

Unopposed ET in women with an intact uterus significantly increases the risk of endometrial cancer. The primary menopause-related indication for progestogen use is endometrial protection from unopposed ET.
Postmenopausal women without a uterus should generally not be prescribed a progestogen with systemic estrogen. Progestogen is generally not indicated when low-dose estrogen is administered locally for vaginal atrophy.

\section{Women's Health Initiative (WHI)}

WHI was a combination of several randomized controlled trials, designed in the early 1990s to examine various strategies for the primary prevention and control of some of the most common causes of morbidity and mortality among healthy post-menopausal women aged 50-79 (mean age 63).

Hormone Therapy Trials (HT): This component examined the effects of combined hormones or estrogen alone on the prevention of coronary heart disease and osteoporotic fractures, and associated risk for breast cancer. Women participating in this component took hormone pills or a placebo (inactive pill) until the Estrogen plus Progestin and Estrogen Alone trials were halted early in July 2002 and March 2004, respectively.

The Women's Health Initiative Memory Study (WHIMS) is an ancillary study to the Women's Health Initiative (WHI). WHIMS was designed to determine the effects of Hormone Therapy (HT) on the development and progression of dementia symptoms in postmenopausal women. This trial too was stopped following the premature termination of the hormone trials.

Million Women Study (MWS): MWS was an observational study of more than one million women in the UK, aged 50 and over. The study looked mainly at how hormone replacement therapy affects a woman's breasts and other aspects of her health. Between 1996 and 2000, women aged between 50-64 (mean age 57) attending breast screening at 66 participating NHS Breast Screening Centres in the UK received a study questionnaire.

\section{Interpreting studies}

It is essential to assess studies critically and not to generically extrapolate the results of a single study or trial to all menopausal women. Points to consider include:

1. The appropriateness of the dose/regimen for the age group studied

2. The preparation and route of administration of therapy since these may have different metabolic effects 
3. The age and health profile of the population studied

4. The relevance of the design and duration of the study, drop out rates and unblinding to the end point examined.

A significant contributor to the outcome from the use of hormones during peri- and postmenopause is the time of initiation of HT use in relation to menopause (final menstrual period). There is a real difference between initiation of HT for symptom relief during perimenopause and initiation of systemic HT use 5,10 , or more years beyond menopause for nonsymptom-related indications.

Evidence-based medicine implies that recommendations should be limited to the women for whom the studies are relevant. Although this goal is ideal in principle, it is impossible in practice, given that there will never be adequate RCTs covering all populations, eventualities, drugs, and regimens.

Many large randomized controlled trials (RCTs) and observational studies of postmenopausal women using HT have been published in recent years. No trial is perfect and no single trial should be used to make public health recommendations.

\section{Pretreatment evaluation}

Before considering any therapeutic regimen, including ET/EPT, all women should have a complete health evaluation, including a comprehensive history and physical examination. Mammography should be performed according to national guidelines and age, but preferably within the previous 12 months before initiation of therapy. Other specific examinations, such as bone densitometry, should be considered on a caseby-case basis.

\section{Vasomotor symptoms}

Treatment of moderate to severe vasomotor symptoms (i.e., hot flushes and night sweats) remains the primary indication for systemic ET and EPT.

\section{Treatment of urogenital symptoms in postmeno- pausal women}

Features of estrogen deficiency in the urogenital systems include vulvar and vaginal atrophy, vaginal dryness, dyspareunia, atrophic vaginitis, soreness, recurrent vaginitis, cystitis from colonic germs, post coital cystitis, nocturia, urinary frequency and urgency. When HT is considered solely for this indication, local (not systemic) vaginal ET is generally recommended.
Randomized controlled trials, albeit limited, have shown that low-dose, local vaginal estrogen delivery is effective and well tolerated for treating vaginal atrophy.

The choice of therapy should be guided by clinical experience and patient preference.

Progestogen is generally not indicated when lowdose estrogen is administered locally for vaginal atrophy.

Data are insufficient to recommend annual endometrial surveillance in asymptomatic women using vaginal ET.

Vaginal ET should be continued for women as long as distressful symptoms remain.

For women treated for nonhormone-dependent cancer, management of vaginal atrophy is similar to that for women without a cancer history. For women with a history of hormone-dependent cancer, management recommendations are dependent upon each woman`s preference in consultation with her oncologist.

\section{Coronary heart disease}

The majority of observational and preclinical studies support the potential benefits of systemic ET/ EPT in reducing coronary heart disease (CHD) risk though some recent RCTs have not.

When analyzed by age and time since menopause at initiation of HT, RCTs are in general agreement with observational studies indicating that HT may reduce CHD risk when initiated in younger and more recently postmenopausal women.

Analysis of the time trend in the EPT arm of the WHI showed a significant interaction between CHD risk and time since initiation of EPT, with an increased risk in the first year after initiation and decreased risk of CHD events in later years of EPT use.

Pending additional data, the use of ET/EPT is not recommended as a single or primary indication for coronary protection in women of any age.

It is important to note that the women enrolled in WHI and HERS initiated EPT more than a decade after menopause on average, and the majority of events that produced this pattern occurred in older women.

\section{Osteoporosis}

There is RCT evidence that HT reduces postmenopausal osteoporotic fractures, including hip fractures, even in women without osteoporosis. 
Extended use of HT is an option in younger women at risk such as premature menopause, when alternate therapies are not appropriate or cause side effects, or when the benefit risk ratio of the extended use of alternate therapies is unknown.

\section{Venous thromboembolism}

Observational studies and RCTs have found a significant increase in the risk of venous thromboembolism (VTE) in postmenopausal women using systemic ET/EPT.

Lower doses of oral estrogens may be safer than higher doses. Growing evidence suggests that women with a prior history of VTE or women who possess factor V Leiden are at increased risk for VTE with HT use. There are limited observational data suggesting lower risks of VTE with transdermal than with oral ET, but there are no RCT data on this subject.

\section{Stroke}

Both ET and EPT appear to increase the risk of ischemic stroke in postmenopausal women, but RCT data have not been fully consistent in this regard. The WHI EPT and ET arms demonstrated an increased risk, whereas some other large trials have not.

Women with prevalent cardiovascular disease (CVD) have a high baseline risk of stroke. No HT regimen should be used for the primary or secondary prevention of stroke, and HT should be particularly avoided for women who have an elevated baseline risk of stroke.

\section{Breast cancer}

Breast cancer risk increases with EPT use beyond 5 years. In absolute terms, this increased risk was rare in the WHI, being 4 to 6 additional invasive cancers per 10,000 women per year who used EPT for 5 or more years.

Women in the ET arm of the WHI demonstrated no increase in risk of breast cancer after an average of 7.1 years of use, with 8 fewer cases of invasive breast cancer per 10,000 women per year of ET use.

Irrespective of the type of HT prescribed, breast cancer risk falls after cessation of use, risk being no greater than that in women who have never been exposed to HT after 5 years.

\section{Endometrial cancer}

Unopposed oestrogen replacement therapy increases endometrial cancer risk. This increased risk persists for several years after ET discontinuation. To negate this increased risk, adequate concomitant progestogen use is recommended for women with an intact uterus. No increased risk of endometrial cancer has been found with continuous combined regimens.

\section{Colorectal cancer}

Results from the oestrogen progestogen arm, but not the oestrogen alone arm, of the WHI study were consistent with data from case control and cohort studies that HRT reduces the risk of colorectal cancer by about a third. However little is known about colorectal cancer risk when treatment is stopped. There is no information about HRT in high risk populations and current data do not allow prevention as a recommendation.

\section{Ovarian cancer}

This issue is unresolved and requires further examination and there is currently insufficient evidence to recommend alterations in HRT prescribing practice

\section{Diabetes mellitus}

Large RCTs suggest that HT reduces new onset of diabetes mellitus (DM).

There is inadequate evidence to recommend combined EPT for a sole indication of the prevention of DM in perimenopausal women. This is a promising area for future research.

\section{Gallbladder disease}

WHI confirmed the observation of the Heart and Estrogen/progestin Replacement Study published in 1998 that HRT increases the risk of gallbladder disease. Gallbladder disease increases with ageing and with obesity, and as confounder HRT users may have silent pre-existing disease.

\section{Dementia and cognitive decline}

HT cannot be recommended at any age for the sole or primary indication of preventing cognitive aging or dementia.

\section{Depression}

Although HT might have a positive effect on mood and behavior, evidence is insufficient to support the use of ET/EPT for the treatment of depression in general. 


\section{Premature menopause and premature ovarian failure}

Premature menopause and premature ovarian failure are conditions associated with a lower risk of breast cancer and earlier onset of osteoporosis and $\mathrm{CHD}$, but there are no clear data as to whether ET or EPT will affect morbidity or mortality from these conditions.

The risks attributable to HT use by these young women receiving HT are likely smaller and the benefits potentially greater than those in older women who commence HT at or beyond the typical age of menopause, although no trial data exist.

\section{Lower doses}

Lower-than-standard doses of ET and EPT should be considered (i.e., daily doses of $0.3 \mathrm{mg}$ oral conjugated estrogens, $0.25-0.5 \mathrm{mg}$ oral micronized 17A-estradiol, $0.025 \mathrm{mg}$ transdermal 17A-estradiol patch, or the equivalent). However, lower doses have not been tested in long-term trials. Some women may require additional local therapy for persistent vaginal symptoms.

\section{Non-oral therapy}

Nonoral routes of administration of ET/EPT may offer advantages and disadvantages, but the long-term risk-benefit ratio has not been demonstrated.

There is some evidence that transdermal 17Aestradiol may be associated with lower risk of deep venous thrombosis than oral estrogen.

\section{Long-term therapy in symptomatic women}

The effects of ET/EPT on risk of breast cancer, $\mathrm{CHD}$, stroke, total CVD, and osteoporotic fracture in perimenopausal women with moderate to severe menopause symptoms have not been established in RCTs.

Data from large studies such as the WHI and HERS should not be extrapolated to symptomatic postmenopausal women younger than 60 years of age who initiate HT at that time as these women were not studied in those trials.

The data should also not be extrapolated to women experiencing premature menopause (?40 y) and initiating $\mathrm{HT}$ at that time.

\section{Symptom recurrence}

Symptoms have an approximate $50 \%$ chance of recurring when therapy is discontinued, independent of age and duration of ET/EPT use. The decision to continue HT should be individualized on the basis of severity of symptoms and current risk-benefit ratio considerations, provided the woman in consultation with her healthcare provider believes that continuation of therapy is warranted.

\section{Indications for extended use}

Extended use of the lowest effective dose for treatment goals of ET or EPT is acceptable under the following circumstances, provided that the woman is well aware of the potential risks and benefits and that there is clinical supervision:

- For the woman for whom, in her own opinion, the benefits of menopause symptom relief outweigh risks, notably after failing an attempt to stop HT.

- For women who are at high risk of osteoporotic fracture and also have moderate to severe menopause symptoms.

- For further prevention of bone loss in the woman with established reduction in bone mass when alternate therapies are not appropriate for that woman or cause side effects or when the outcomes of the extended use of alternate therapies are unknown.

\section{Quality of life}

An improvement in health-related QOL (HQOL) can result with HT through decreased menopausal symptoms and possible elevation of mood that leads to a feeling of well-being.

While some studies have shown improvement in both symptomatic and asymptomatic women, others have not. This lack of effect seen in WHI is hardly surprising, since the study participants were largely asymptomatic. This area is difficult to evaluate because of the different measures used, varying levels of menopausal symptomatology, a large placebo effect and extrinsic factors which may alter women's responses.

\section{Summary of the position statement of the Menopause Society of Sri Lanka}

The results from WHI and MWS are not necessarily relevant to younger postmenopausal women taking appropriate doses of different regimens

Giving one HT regimen to women in WHI who had no particular indication to take it resulted in neither harm nor benefit to over $99 \%$. 
The oestrogen alone arm of WHI found a reduced risk of breast cancer and coronary heart disease in women less than 60 years of age.

HT should currently not be prescribed solely for possible prevention of cardiovascular disease and dementia.

The results from recent papers have thus far given no reason to make any change in current clinical practice for the use of HT

\section{Summary practice points}

(i) Most women who request HT do so for symptom relief which is the main indication for HT use in peri and postmenopausal women. Treatment for up to five years does not add significantly to lifetime risk of breast cancer, but significantly decreases bone loss and risk of osteoporotic fractures.

(ii) Women with a premature menopause should normally be offered HT until the average age of the menopause (52 years)

(iii) Some women may be susceptible to early thrombotic risk, but when appropriate HRT is given after individual clinical evaluation, the benefits will far outweigh any potential risks and the treatment should be recommended.

(iv) The merits of long term use need to be assessed for each individual at regular intervals, such as annually

(v) Women whose uterus is present should be offered appropriate progestogen therapy as oestrogen alone will increase the risk of endometrial cancer

\section{Alternative and complementary therapies}

\section{Tibolone}

Tibolone is a synthetic steroid that has oestrogenic, progestogenic and androgenic properties. It is effective in treating menopausal symptoms. It conserves bone mass, and preliminary data have demonstrated a reduction in vertebral fractures. Further fracture data will be forthcoming.

Tibolone shares some of the risks of oestrogen based HRT. The MWS showed an increased risk of breast cancer and endometrial cancer. However, it is probable that the level of risk is overestimated in this study and the actual level is uncertain. Again data from randomised controlled trials are awaited.

\section{Phytoestrogens}

Phytoestrogens are plant substances that have effects similar to those of estrogens. Preparations vary from enriched foods, such as bread or drinks (e.g. soy milk), to more concentrated tablets.

The most important groups are called isoflavones and lignans.

The major isoflavones are genistein and daidzein. The major lignans are enterolactone and enterodiol.

Isoflavones are found in soybeans, chickpeas, red clover and probably other legumes (beans and peas).

Lignans are found in oil seeds such as flaxseed, and they also are found in cereal bran, whole cereals, vegetables, legumes and fruit.

The role of phytoestrogens has stimulated considerable interest, as people from populations that consume a diet high in isoflavones, such as the Japanese, are claimed to have lower rates of: menopausal vasomotor symptoms; cardiovascular disease; osteoporosis; and breast, colon, endometrial and ovarian cancers.

With regard to menopausal symptoms, the evidence from randomized placebo-controlled trials in Western populations is conflicting for soy and derivatives of red clover. Similarly, debate also surrounds the effects on lipoproteins, endothelial function, blood pressure, cognition, the endometrium and the breast.

It is clear that foods or supplements that contain isoflavones have some physiologic effects. Clinicians may wish to recommend that menopausal women consume whole foods that contain isoflavones, especially for the cardiovascular benefits of these foods; however, a level of caution needs to be observed in making these recommendations.

Further well designed, randomized clinical trials are needed before specific recommendations can be made regarding increased consumption of foods or supplements that contain high amounts of isoflavones and to determine the role and safety of phytoestrogen supplements in perimenopausal and postmenopausal women.

\section{Herbalism}

Herbal remedies need to be used with caution in women with a contraindication to estrogen, as some herbs have estrogenic properties. Herbs used by menopausal women include: 
- Actaea racemosa (black cohosh)

- Piper methysticum (kava kava)

- Oenothera bienis (evening primrose)

- Angelica sinensis (dong quai)

- Ginkgo biloba (ginkgo)

- Panax ginseng (ginseng)

- Others - wild yam cream, St John's wort, Vitex agnus-castus, liquorice root, Valerian root.

Interaction with conventional therapies is a major concern as the consequences are potentially fatal.

The consequences of herb-drug interactions include:

- Bleeding when combined with warfarin or aspirin

- Hypertension, coma and mild serotonin syndrome when combined with serotonin reuptake inhibitors

- Reduced efficacy of antiepileptics.

- Reduces the blood concentrations of cyclosporin, midazolam, tacrolimus, amitriptyline, digoxin, warfarin and theophylline

- There is also concern about interactions with anticancer drugs.

Furthermore, little control over the quality of the products exists, so it is unusual to know what is actually present in individual herbal preparations and dietary supplements.

Severe adverse reactions, including renal and liver failure and cancer, have been reported. Moreover, some preparations contain high levels of heavy metals, such as arsenic, lead and mercury.

Liver toxicity has been reported with black cohosh.

\section{Summary practice points}

(i) Evidence from randomized trials that alternative and complementary therapies improve menopausal symptoms or have the same benefits as hormone replacement therapy is poor.

(ii) There is concern about interactions with other treatments, as these may have potentially fatal consequences.

(iii) Some preparations may contain estrogenic compounds and this is a concern for women with hormone-dependent diseases.

\section{The role of testosterone therapy in postmenopausal women}

Endogenous testosterone levels have not been clearly linked to sexual function in postmenopausal women. Published evidence from randomized controlled trials, although limited, indicates that exogenous testosterone, both oral and nonoral formulations, has a positive effect on sexual function, primarily desire, arousal, and orgasmic response, in women after spontaneous or surgically induced menopause.

Postmenopausal women with decreased sexual desire associated with personal distress and with no other identifiable cause may be candidates for testosterone therapy.

Testosterone treatment without concomitant estrogen therapy cannot be recommended because of a lack of evidence.

When evaluating a woman for testosterone therapy, recommendations are to rule out causes not related to testosterone levels (eg, physical and psychosocial factors, medications) and to ensure that there is a physiologic cause for reduced testosterone levels (eg, bilateral oophorectomy).

Laboratory testing of testosterone levels should be used only to monitor for supra-physiologic levels before and during therapy, not to diagnose testosterone insufficiency.

Monitoring should also include subjective assessments of sexual response, desire, and satisfaction as well as evaluation for potential adverse effects.

Transdermal patches and topical gels or creams are preferred over oral products because of first-pass hepatic effects documented with oral formulations.

Testosterone products formulated specifically for men have a risk of excessive dosing, although some clinicians use lower doses of these products in women.

Testosterone therapy is contraindicated in women with breast or uterine cancer or in those with cardiovascular or liver disease.

It should be administered at the lowest dose for the shortest time that meets treatment goals.

Counselling regarding the potential risks and benefits should be provided before initiating therapy. 
The role of calcium in peri- and post-menopausal women

The most definitive role for calcium in peri- and postmenopausal women is in bone health, but, like most nutrients, calcium has beneficial effects in many body systems. Based on the available evidence, there is strong support for the importance of ensuring adequate calcium intake in all women, particularly those in peri- or post-menopause.

\section{References}

1. British Menopause Society: Consensus Statement on Hormone replacement therapy 27 June 2007.

2. European Menopause and Andropause Society (EMAS) 2004/ 2005 position statements on peri- and post-menopausal hormone replacement therapy. Maturitas 2005; 51.

3. Menopause: The Journal of The North American Menopause Society 2005; 12: 496.

4. Menopause: The Journal of The North American Menopause Society 2008; 15: 584-603. 